\title{
Domains of outcomes in spinal cord injury for clinical trials to improve neurological function
}

\author{
Ralph J. Marino, MD, MSCE \\ Department of Rehabilitation Medicine, Jefferson Medical College, Thomas Jefferson University, Philadelphia, PA
}

\begin{abstract}
Selecting appropriate outcome measures in spinal cord injury clinical trials that aim to improve spinal cord function is a challenging task. Proving that an intervention has the intended effect on neurological functioning is insufficient. Any improvement must be shown to be clinically significant-that is, makes a change in the life of the person. Rehabilitation, which seeks to maximize function despite residual impairments, complicates this task. Small improvements in function due to a spinal cord intervention may be masked by improvements in function due to rehabilitation, particularly in the initial rehabilitation phase of care. Understanding outcome domains in models of disablement will facilitate selection or development of assessment tools appropriate for the trial in question. This article describes an expanded World Health Organization model of functioning that defines the domains of Impairment, Capability/Functional Limitation, and Activity, as well as the subdomains of Capacity and Performance, and gives examples of measurement instruments in use or under development in each domain.
\end{abstract}

Key words: activities of daily living, disability model, functional limitation, ICF, impairment, measurement, participation, rehabilitation, spinal cord injury, spinal cord intervention.

\section{INTRODUCTION}

This article provides a conceptual framework for assessing outcomes in spinal cord injury (SCI) clinical trials that aim to improve neurological function. The information in this article was presented as an introduction to a 1-day course on outcome assessment in SCI that was held May 11, 2005, in Dallas, Texas. The content is limited to those measurement instruments and relevant domains presented at the course and is not meant to be a comprehensive review of domains and instruments in SCI.

Clinical trials in SCI, particularly trials in the acute stage of injury that evaluate interventions for preserving or improving spinal cord function, are conducted against the backdrop of rehabilitation. In addition to demonstrating an effect on neurological functioning, investigators in such clinical trials are encouraged to demonstrate that any improvement is clinically significant-that is, results in improvements in functional tasks and social participation. The second National Acute Spinal Cord Injury study, which found that a group receiving methylprednisolone within 8 hours of injury had improved motor scores compared with a placebo group [1], was criticized for not including a functional outcome measure. Demonstrating improved functioning at the level of functional

Abbreviations: ASIA = American Spinal Injury Association; FIM = Functional Independence Measure; ICF = International Classification of Functioning, Disability, and Health; ICIDH = International Classification of Impairments, Disabilities, and Handicaps; ICSHT = International Classification for Surgery of the Hand in Tetraplegia; IOM = Institute of Medicine; ISCoS = International Spinal Cord Society; MRI = magnetic resonance imaging; QIF = Quadriplegia Index of Function; SCI = spinal cord injury; SCIM = Spinal Cord Independence Measure; WHO = World Health Organization; WISCI = Walking Index for Spinal Cord Injury.

Address all correspondence to Ralph J. Marino, MD, MSCE; 132 South 10th Street, Suite 375 Main, Philadelphia, PA 19107; 215-955-5756; fax: 215-955-5152.

Email: ralph.marino@jefferson.edu

DOI: 10.1682/JRRD.2005.08.0138 
activities or social participation in such a study can be difficult in patients undergoing rehabilitation, because the rehabilitation process seeks to minimize the impact of impairments on functioning [2]. For example, orthoses, assistive devices, wheelchairs, and environmental modifications may be provided to help a person with a disability get from one place to another. The more successful these adaptations are, the weaker the relationship between impairment and activity will be and the harder it will be to show that the experimental treatment made a difference [3]. On the Functional Independence Measure $\left(\mathrm{FIM}^{\mathrm{TM}}\right)$, for instance, the same locomotion score is given to a person who walks independently with a walker and bilateral orthoses as is given to a person who walks with a cane and no braces. An impairment change that allows a person with paraplegia to trade in his or her braces and walker for a cane will not be reflected in that person's FIM locomotion score.

Selecting an appropriate measurement instrument is facilitated by an understanding of domains of outcomes and their interrelationships. The conceptualization of disablement has been the subject of numerous publications and different models have been proposed by the Institute of Medicine (IOM) [2,4], the National Center for Medical Rehabilitation Research [5], and the World Health Organization (WHO) [6-7]. This article will use the International Classification of Functioning, Disability, and Health (ICF) (Figure 1) [7], which is the basic WHO framework, with an additional domain (Capability/Functional Limitation) inserted between the Body Functions and Structures and Activity domains.

\section{MODEL OF FUNCTIONING AND DISABILITY}

The ICF is a revision of the International Classification of Impairments, Disabilities, and Handicaps (ICIDH) [6] and better reflects the complex relationship between illness or disease and functioning. The ICF model distinguishes between the domains of (1) Body Functions and Structures, (2) Activities, and (3) Participation and includes two sets of contextual factors, Environmental and Personal (Figure 1). A health condition may affect any one of the three domains. The ICF is more neutral than the ICIDH and focuses on human functioning rather than disability alone. Domains may be described in either positive or negative terms, such as "activity” versus "activity limitation.”

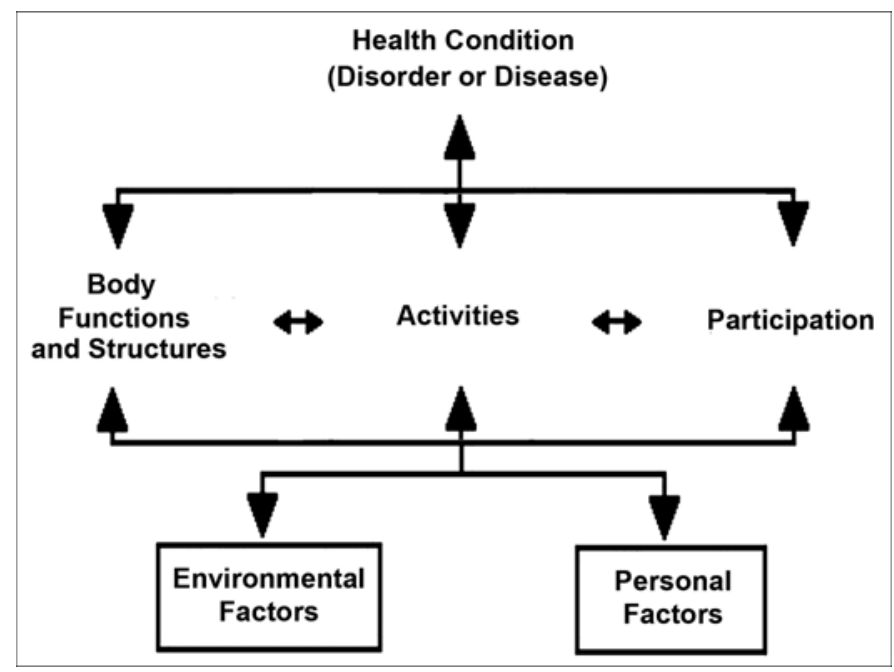

Figure 1.

World Health Organization (WHO) International Classification of Functioning, Disability, and Health model of functioning. Reprinted by permission from WHO. WHO. International Classification of Functioning, Disability, and Health. 2001. p. 18.

In the Body Functions and Structures domain, body functions are physiological functions of body systems, such as sensation and muscle power [7]. Body structures are the anatomical parts of the body, such as organs and limbs and their components. The negative descriptor for these terms is "impairment," defined as a "problem in body function or structure as a significant deviation or loss" [7].

The Activity domain requires the use of qualifiers of functioning, namely capacity and performance, which are hereafter referred to as subdomains. Activity is the execution of a task or action by an individual, and activity limitations are difficulties in executing activities [7]. The Capacity subdomain describes a person's ability to execute a task or action. It documents a person's highest level of functioning in a given environment and requires testing in a standardized environment. This standardized environment is often more, but may be less, accommodating than the person's usual environment. The Performance subdomain, on the other hand, describes what a person routinely does in his or her current environment. This environment may differ from individual to individual and from one time to another.

The Participation domain describes an individual's involvement in a life situation, such as work, school, or sports, and requires performance of various actions and tasks. The ICF contains a single taxonomic list of items for both the Activity and Participation domains and 
indicates that the Capacity subdomain applies only to the Activity domain and that the Participation domain should indicate performance [7]. The contextual factors, which may influence functioning, are grouped into the domains of Environmental Factors and Personal Factors and may be facilitators of or barriers to function. Environmental factors are aspects of the physical, social, and attitudinal environment. Personal factors are the myriad features of an individual's life that influence functioning, such as age, coping style, education, and social background.

Nagi thought that the ICIDH lacked "a coherent and clearly delineated concept of [functioning] at the level of the organism" [8]. He proposed the domain of Functional Limitation to fill this gap. As incorporated into the IOM model of disablement, functional limitation is defined as a "restriction or lack of ability to perform an action or activity in the manner or within the range considered normal" [2]. Unfortunately, certain terms, such as "action" and "activity," are defined differently by the WHO and the IOM. For clarity in this article, Capability/Functional Limitation items will be called "actions" and the ability to perform actions "capability." The terms "tasks" and "activities" will be reserved for items in the Activity domain and its Capacity and Performance subdomains. Functional limitations operate at the person level and represent the total effect of all impairments on actions. Items that could be considered functional limitations, such as sitting or grasping, are included in the Activity taxonomy of the ICF but are not identified as such. The modified ICF model that we use in the rest of this article is depicted in Figure 2. This model extracts the Capability/ Functional Limitation domain from the Activity domain and explicitly divides the Activity domain into the subdomains of Capacity and Performance. As will be explained, separating Capability/Functional Limitation from Capacity Limitation from Performance Limitation makes sense conceptually when one is determining the impact of improvement in impairment on functioning.

The complexity of measurement increases as one moves through the domains from Body Functions and Structures/Impairment (hereafter referred to as Impairment) to Participation because more and more factors may influence function at each successive level. The Table delineates the similarities and differences among the domains of Impairment, Capability/Functional Limitation, Activity, and Participation. For example, strength of the finger flexor or thenar muscles would fall into the Impairment domain. A capability/functional limitation related to

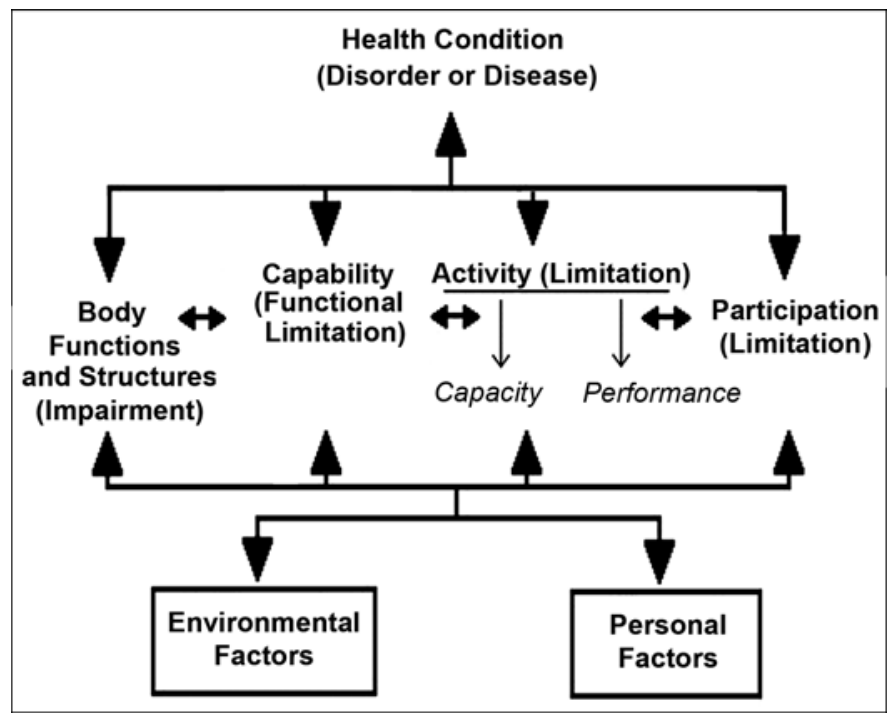

Figure 2.

Modified International Classification of Functioning, Disability, and Health model of functioning. Model extracts Capability/Functional Limitation from Activity Limitation and explicitly divides Activity into Capacity and Performance subdomains.

finger flexor strength would be the ability to grasp a cylindrical object, such as a glass of water or a can of soup. The exact object is not critical but rather the type of grasping pattern it represents, in this case cylindrical grasp as opposed to pinch or key grip. Grasping ability may be affected by impairments other than weakness, such as spasticity or contractures. The Capacity subdomain focuses on tasks or activities, not the action of the body. Ideally, but not necessarily, when capacity is measured, the patient should perform the task or activity without equipment or assistance; this differs from measurement of capability/functional limitation, where neither equipment nor assistance is allowed. In determining the individual's capacity to grasp and lift a glass of water, the object is important and should be standardized, as should the decision to allow equipment or assistance. Picking up a glass without a handle is different from lifting a cup with a handle. The Performance subdomain, on the other hand, focuses on what happens in everyday situations. The object may be modified to facilitate performance; for example, a cup with a handle may be used, equipment such as a wrist-driven orthosis may be provided and, if necessary, physical assistance may be provided. The relevant area of participation in this example would be dining out with friends, during which getting a drink is part of the set of activities performed. The method of getting a drink 
Table.

Characteristics of domains of outcome in modified International Classification of Functioning, Disability, and Health model.

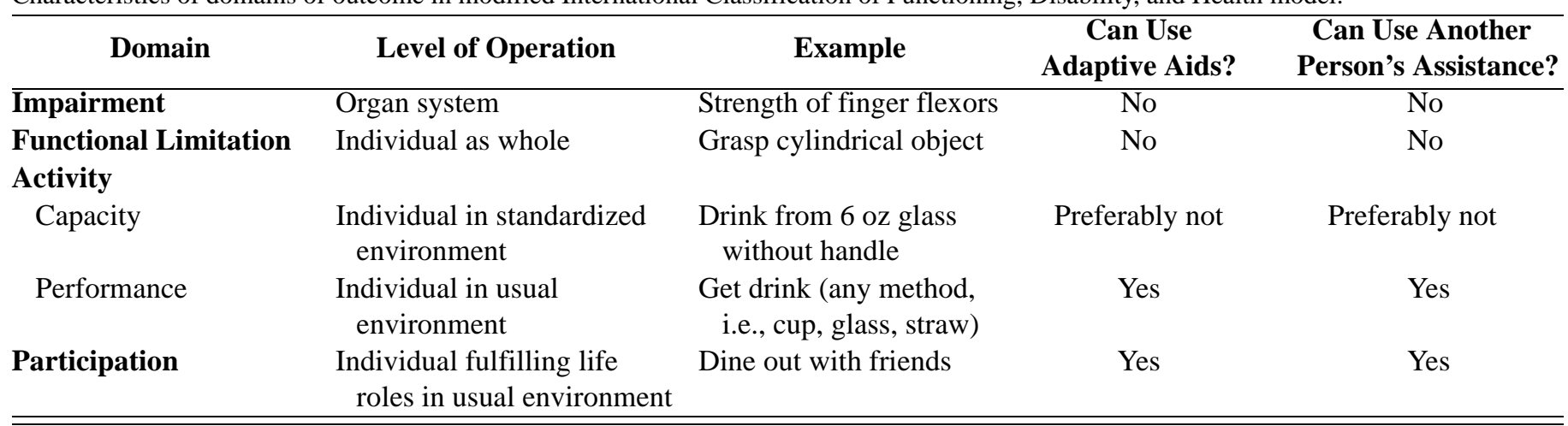

is much less important here, so use of a long straw to eliminate the need to grasp or lift a glass is a valid method for accomplishing the activity. At the level of Participation, then, the ability to get a drink (after set up) has little relationship to finger flexor strength or the ability to grasp an object.

Evaluating functional limitations during a clinical trial of an agent targeting neurological function can substantiate the real-life benefit of the intervention by demonstrating that the treated subjects can do more than control subjects; i.e., they can interact with the environment in ways that the control subjects cannot. Impairment measures can be somewhat abstract and the clinical relevance of changes unclear. As Walker stated in an editorial on a clinical trial in acute SCI, "clinicians wonder ... what a 'seven-point change' in the ASIA [American Spinal Injury Association] score really means” [9]. The ICF includes capacity and performance with and without devices and/or assistance in a single domain, and this blurs important distinctions among these ways of functioning. A very strict interpretation of the ICF conceptualization of capacity would approximate evaluation of functional limitations. However, most instruments that measure activity do not require specification of the type of orthosis or adaptive device, only whether or not one is used. Furthermore, although adaptive equipment is part of the ICF Environmental Factor domain, valid objective measurement instruments for environmental factors are not widely available.

During a clinical trial in which the intervention targets the structure and function of the spinal cord itself, such as growth-promoting factors or cell transplants, detecting improvements at the levels of Activity (Performance) or Participation may be difficult. As one moves further away from the domain targeted by the intervention, detecting changes becomes harder, because environmental and personal factors and comorbid conditions grow in importance. The effect that is quite large at the level of Impairment may be immeasurable at the level of Participation [3]. Persons with the same SCI level and severity may have very different levels of activity performance because of differences in availability of adaptive equipment and personal assistance, accessibility of the environment, and expectations of family members. The contextual factors often supersede impairments in their influence on participation. Impairment is not highly correlated with Participation [10]. The inability to walk does not prevent one from working nor does a weak grasp prevent one from eating out.

A thorough understanding of the mechanism of the intervention and its effect on impairment coupled with knowledge of a model of disablement like the one presented here, may facilitate selection of an Activity instrument targeted to the intervention. Ultimately, a battery of instruments that focuses on several domains of function is desirable. Demonstrating improvement in the Activity subdomains of Capacity and Performance expected by changes seen in the Impairment and Capability/Functional Limitation domains would support the premise that the intervention caused the improvements. Conversely, improvement in measures of activity without a corresponding improvement in impairment or capability/functional limitation would suggest that other factors, for example, task modification or use of equipment, caused the improvement. Following are examples of instruments used in SCI research across the domains of the modified model of disablement. Since the focus is clinical trials that target impairment, which is not a major determinant 
of participation, participation outcome instruments will not be addressed.

\section{IMPAIRMENT: TISSUES AND CELLS}

Efforts are underway to better assess the pathology or altered functioning of the spinal cord in human SCI. The ICF currently does not contain a classification for alterations at the tissue and cellular levels, such as the spinal cord, but allows for its inclusion in the future [7, p. 12] Techniques for evaluating spinal cord tract function are under development, however. Somatosensory- and motor-evoked potentials have been somewhat successful in predicting recovery after SCI [11-13]. To date, these measures have not predicted recovery more accurately than the clinical neurological examination [12]. In another line of investigation, researchers sponsored by the International Spinal Research Trust have been developing other physiological tests of spinal cord tract function [14]. They have described advances in measures of motor function that use transcranial magnetic stimulation and recording over limb and trunk muscles [15-16]. This work is yielding insight into muscle function in the thoracic segments of the spinal cord, where clinical testing of muscles is quite limited.

Advances in magnetic resonance imaging (MRI) enable us to see the injured spinal cord in greater detail. MRI evaluates "body structure" and alterations in that structure. Attempts have been made to relate findings on MRI to current and future neurological function [17-18]. Abnormalities found to correlate with neurological function include presence of hemorrhage, edema, and length of edema in the spinal cord [19]. Functional MRI is a technique that merits attention. In the future, it may help us evaluate spinal cord function. This technique was useful in assessing spinal cord response to a thermal stimulus, whether or not the patient was aware of the stimulus [20].

\section{IMPAIRMENT: BODY FUNCTIONS AND STRUCTURES}

In SCI, the most widely used and accepted impairment instrument is the International Standards for Neurological Classification of Spinal Cord Injury [21], developed by ASIA and the International Spinal Cord Society (ISCoS). Sensory and motor functions are evalu- ated at the bedside with standardized techniques and minimal equipment [22]. Interrater reliability of the total sensory scores and motor scores is very good overall (intraclass correlation coefficients $>0.96$ ), although values are lower for subgroups with incomplete injuries [22-23]. Agreement in classification, which is a different skill, has improved with successive revisions of the International Standards and, among experienced clinicians, is now as high as 93 to 94 percent for motor and sensory levels and 87 percent for ASIA impairment scale grade [23].

In the International Standards, sensory and motor functions are measured by ordinal scales and more interval-level measures of impairment have been desired. Current work is developing more quantitative measures of sensory function. Aspects of sensory testing being evaluated include light-touch threshold (using SemmesWeinstein monofilaments), vibration perception (using a bio-thesiometer), and thermal perception and pain thresholds [14]. In the area of motor function, hand-held dynamometry offers continuous-level measurement of muscle power as an alternative to ordinal-level manual muscle strength testing. Some evidence exists that hand-held dynamometry is more sensitive to change than manual muscle testing [24] and is better correlated to activities [25]. Additional research must determine whether handheld dynamometry is useful for evaluating motor function in clinical trials.

The International Standards are not the only classification system used in SCI care and research. Surgeons involved in upper-limb reconstructive procedures have found that the classification of motor levels in the International Standards is too broad. Instead of the 5 motor levels from the fifth cervical to the first thoracic, these surgeons have developed a 10-level scale called the International Classification for Surgery of the Hand in Tetraplegia (ICSHT). It is based on the number of muscles below the elbow available for tendon transfer. At each successive level, an additional specified muscle with manual muscle test score of at least grade 4 must be available for tendon transfer [26]. The ICSHT is the accepted system for classification of patients when results of upper-limb reconstructive procedures in SCI are being reported.

Autonomic function is another important area that is not addressed by the International Standards. Evaluation of autonomic functions has been plagued by lack of a common terminology. In an attempt to fill this void, ASIA and ISCoS have formed a committee that will draft 
a classification system for autonomic functions. The committee is divided into four subcommittees: (1) general autonomic functions, such as blood pressure, heart rate, and temperature regulation; (2) bladder function; (3) bowel function; and (4) sexual function. Krassioukov et al. present the committees' preliminary conceptualizations and classifications of autonomic functions and welcome comments and suggestions [27].

\section{CAPABILITY/FUNCTIONAL LIMITATION}

Given the lack of consensus in the field over the place of "functional limitations" in disablement models, one should not be surprised that measures of this domain are limited in number. Most available measures focus on the upper limb. While many measurement instruments for hand function are available, only a few have been used for the evaluation of interventions in SCI. One such measure is the Grasp and Release Test that evaluates the functional impact of an implanted upper-limb neuroprosthesis [28]. This test, as the name implies, focuses on the grasping functions of the hand. A more comprehensive assessment of upper-limb function is the Capabilities of Upper Extremity instrument [29]. This instrument evaluates actions of the arm, such as reaching and pushing, and of the hand, such as grasping and manipulating objects. Details of this instrument, which is still under development, can be found elsewhere [23]. It has been used to document changes in capabilities of the arms and hands after tendon transfer and neuroprosthesis implantation [30]. In a small series of patients, the Capabilities of Upper Extremity instrument accurately differentiated improvements based on whether the procedures targeted the arm, the hand, or both [30].

\section{ACTIVITY: CAPACITY}

The next area of interest is capacity, i.e., what a person can do in a standardized environment [7]. Lack of proper equipment or an inaccessible environment may limit a person's performance of an activity even if he or she has the capacity to complete it. To minimize confounding by environmental differences, one must assess activities in a standardized environment, that is, assess capacity. In SCI, capacity measures have focused on assessment of walking function. Walking is commonly evaluated by tests that measure time to walk a certain distance, such as the $10 \mathrm{~m}$ walk or the Timed Get-up-andGo test, or the distance walked in a certain time, such as the 6-minute walk test [31]. In addition to time and distance measurements, any equipment and/or assistance that the person uses when walking must be recorded. A validated instrument for this purpose is the Walking Index for Spinal Cord Injury (WISCI), a hierarchical scale that ranks walking function based on various combinations of braces, assistive devices, and levels of personal assistance [32]. Because use of equipment and physical assistance is permitted, the WISCI does not fall into the Capability/Functional Limitation domain. However, higher levels represent lesser degrees of impairment, not independence, so the ranking differs from that of most activity instruments.

\section{ACTIVITY: PERFORMANCE}

The Performance subdomain encompasses what an individual actually does at a given time in his or her usual or current environment. When evaluating performance, the therapist aims to reflect the person's actual life situation [7]. Therefore, if personal assistance or assistive devices are typically used to accomplish an activity, then they are used when the activity is rated. Performance is generally what is measured during inpatient rehabilitation programs. In the United States, the most often used instrument is the FIM, a generic instrument that assesses burden of care of patients undergoing inpatient rehabilitation [33]. FIM scores may overestimate performance if therapists rate patients based on what they do during therapy sessions rather than what they do outside of therapy. In addition, performance may decline after discharge if the discharge environment is not as accommodating as the rehabilitation unit.

As do most generic measurement instruments, the FIM has shortcomings when applied to a specific condition such as SCI. Dissatisfaction with the FIM has led to more condition-specific instruments. In SCI, such measurement tools include the Quadriplegia Index of Function (QIF) [34] and the Spinal Cord Independence Measure (SCIM) [35]. The QIF is limited to use in tetraplegia and has been used sparingly, while the SCIM is gaining in popularity. Both instruments take a similar approach as the FIM but contain items and levels that are more relevant to SCI. For example, the QIF feeding category 
separately rates tasks, such as pouring liquids, applying spreads, and opening containers, that have different degrees of difficulty for patients with tetraplegia, while the FIM includes all of these tasks in the "supervision/ set-up” level. Therefore, the QIF feeding category is more highly correlated than the FIM eating category with upper-limb motor scores in tetraplegia [36]. Unlike the FIM, which only addresses indoor mobility, the SCIM separately evaluates indoor and outdoor mobility and lists both wheelchair use and walking as possible levels of functioning [37].

Another approach to evaluation of performance is individualized assessment, as has been used in the Canadian Occupational Performance Measure [38]. This client-centered performance tool is used by occupational therapists. It requires a semistructured interview for identification of client desires for change in the areas of selfcare, productivity, and leisure. The five most important areas (to the client) are selected and tracked. At the start and completion of treatment, the client rates these five areas for performance and satisfaction with performance using a 1 to 10 scale. Scores are summed and averaged over the number of areas and mean performance and satisfaction scores are obtained. A change in the mean score of 2 or more is considered significant [39]. This approach may have promise in clinical trials, but the choice of problem areas may need to be limited to activities that the intervention is expected to affect.

\section{CONCLUSIONS}

This article presented a framework for evaluating changes in impairment, capability/functional limitation, and activity limitation in SCI clinical trials targeting spinal cord function. An understanding of the domains of outcome and their interrelationships allows selection of appropriate outcome measurement instruments in clinical trials. A battery of instruments targeting the different domains of function from Impairment to Performance should be used for full evaluation of an intervention. Measurement instruments that evaluate the Impairment domain to demonstrate that the intervention does what it is intended to do should be emphasized. Achieving improvement in the Capability/Functional Limitation and Activity domains will provide evidence that Impairmentdomain changes are "clinically" significant. However, as one assesses functioning in more distant domains, the link between the intervention and outcome will weaken. For Impairment-targeted interventions, functional impact will be identified more easily with measures of Capability/Functional Limitation or Capacity than with measures of Performance.

\section{ACKNOWLEDGMENTS}

This material was based on work supported by the National Institute on Disability and Rehabilitation Research, Office of Special Education and Rehabilitative Services, U.S. Department of Education, grant H133N000023 to the Regional Spinal Cord Injury Center of the Delaware Valley.

The authors have declared that no competing interests exist.

\section{REFERENCES}

1. Bracken MB, Shepard MJ, Collins WF, Holford TR, Young W, Baskin DS, Eisenberg HM, Flamm E, Leo-Summers L, Maroon JC, Marshall LF, Perot PL, Piepmeier J, Sonntag VKH, Wagner FC, Wilberger JE, Winn HR. A randomized, controlled trial of methylprednisolone or naloxone in the treatment of acute spinal cord injury. Results of the Second National Acute Spinal Cord Injury Study. N Engl J Med. 1990;322(20):1405-11. [PMID: 2278545]

2. Brandt EN Jr, Pope AM, editors. Enabling America: Assessing the role of rehabilitation science and engineering: Executive summary. Washington (DC): National Academy Press; 1997.

3. Whyte J. Toward a methodology for rehabilitation research. Am J Phys Med Rehabil. 1994;73(6):428-35.

[PMID: 7993617]

4. Pope AM, Tarlov AR, editors. Disability in America: Toward a national agenda for prevention. Washington (DC): National Academy Press; 1991.

5. Research plan for the National Center for Medical Rehabilitation Research. NIH Publication No. 93-3509. Washington (DC): U.S. Department of Health and Human Services; 1993.

6. Wood P. International classification of impairments, disabilities, and handicaps. Geneva (Switzerland): World Health Organization; 1980.

7. World Health Organization. International classification of functioning, disability and health: ICF. Geneva (Switzerland): World Health Organization; 2001.

8. Nagi SZ. Disability concepts revisited: Implications for prevention. In: Pope AM, Tarlov AR, editors. Disability in America: Toward a national agenda for prevention. Washington (DC): National Academy Press; 1991. p. 309-27. 
9. Walker MD. Acute spinal-cord injury. N Eng J Med. 1991; 324(26):1885-87. [PMID: 1904134]

10. Whiteneck GG. Outcome evaluation and spinal cord injury. NeuroRehabilitation. 1992;2(4):31-41.

11. Curt A, Dietz V. Electrophysiological recordings in patients with spinal cord injury: Significance for predicting outcome. Spinal Cord. 1999;37(3):157-65. [PMID: 10213324]

12. Iseli E, Cavigelli A, Dietz V, Curt A. Prognosis and recovery in ischaemic and traumatic spinal cord injury: Clinical and electrophysiological evaluation. J Neurol Neurosurg Psychiatry. 1999;67(5):567-71. [PMID: 10519858]

13. Curt A, Keck ME, Dietz V. Functional outcome following spinal cord injury: Significance of motor-evoked potentials and ASIA scores. Arch Phys Med Rehabil. 1998;79(1):81-86. [PMID: 9440423]

14. Ellaway PH, Anand P, Bergstrom EM, Catley M, Davey NJ, Frankel HL, Jamous A, Mathias C, Nicotra A, Savic G, Short D, Theodorous S. Towards improved clinical and physiological assessments of recovery in spinal cord injury: A clinical initiative. Spinal Cord. 2004;42(6):325-37. [PMID: 14968107]

15. Cariga P, Catley M, Nowicky AV, Savic G, Ellaway PH, Davey NJ. Segmental recording of cortical motor evoked potentials from thoracic paravertebral myotomes in complete spinal cord injury. Spine. 2002;27(13):1438-43. [PMID: 12131743]

16. Davey NJ, Smith HC, Wells E, Maskill DW, Savic G, Ellaway PH, Frankel HL. Responses of thenar muscles to transcranial magnetic stimulation of the motor cortex in patients with incomplete spinal cord injury. J Neurol Neurosurg Psychiatry. 1998;65(1):80-87. [PMID: 9667566]

17. Flanders AE, Schaefer DM, Doan HT, Mishkin MM, Gonzalez CF, Northrup BE. Acute cervical spine trauma: Correlation of MR imaging findings with degree of neurologic deficit. Radiology. 1990;177(1):25-33. [PMID: 2399326]

18. Flanders AE, Spettell CM, Tartaglino LM, Friedman DP, Herbison GJ. Forecasting motor recovery after cervical spinal cord injury: Value of MR imaging. Radiology. 1996; 201(3):649-55. [PMID: 8939210]

19. Schaefer DM, Flanders A, Northrup BE, Doan HT, Osterholm JL. Magnetic resonance imaging of acute cervical spine trauma. Correlation with degree of neurologic injury. Spine. 1989;14(10):1090-95. [PMID: 2588058$]$

20. Stroman PW, Kornelsen J, Bergman A, Krause V, Ethans K, Malisza KL, Tomanck B. Noninvasive assessment of the injured human spinal cord by means of functional magnetic resonance imaging. Spinal Cord. 2004;42(2):59-66. [PMID: 14765137]

21. American Spinal Injury Association. International standards for neurological classification of spinal cord injury, revised 2000. Chicago (IL): American Spinal Injury Association; 2002.
22. American Spinal Injury Association. Reference manual for the international standards for neurological classification of spinal cord injury. Chicago (IL): American Spinal Injury Association; 2003.

23. Marino RJ. Neurological and functional outcomes in spinal cord injury: Review and recommendations. Top Spinal Cord Inj Rehabil. 2005;10(4):51-64.

24. Schwartz S, Cohen ME, Herbison GJ, Shah A. Relationship between two measures of upper extremity strength: Manual muscle test compared to hand-held myometry. Arch Phys Med Rehabil. 1992;73(11):1063-68. [PMID: 1444773]

25. Marciello MA, Herbison GJ, Ditunno JF Jr, Marino RJ, Cohen ME. Wrist strength measured by myometry as an indicator of functional independence. J Neurotrauma. 1995; 12(1):99-106. [PMID: 7783236]

26. McDowell CL, Moberg EA, House JH. The second international conference on surgical rehabilitation of the upper limb in traumatic quadriplegia. J Hand Surg. 1986;11(1):604-8.

27. Krassioukov AV, Karlsson AK, Wecht JM, Wuermser LA, Mathias C, Marino R. Assessment of autonomic dysfunction following spinal cord injury: Rationale for additions to International Standards for Neurological Assessment. J Rehabil Res Dev. 2007;44(1):103-112.

28. Wuolle KS, Van Doren CL, Thrope GB, Keith MW, Peckham PH. Development of a quantitative hand grasp and release test for patients with tetraplegia using a hand neuroprosthesis. J Hand Surg (Am). 1994;19(2):209-18. [PMID: 8201183]

29. Marino RJ, Shea JA, Stineman MG. The Capabilities of Upper Extremity instrument: Reliability and validity of a measure of functional limitation in tetraplegia. Arch Phys Med Rehabil. 1998;79(12):1512-21. [PMID: 9862292]

30. Marino RJ, Mulcahey MJ. Use of the Capabilities of Upper Extremity instrument to evaluate outcomes after upper extremity reconstruction in tetraplegia. J Spinal Cord Med. 2004;27(Suppl 1):S125.

31. Van Hedel HJ, Wirz M, Dietz V. Assessing walking ability in subjects with spinal cord injury: Validity and reliability of 3 walking tests. Arch Phys Med Rehabil. 2005;86(2): 190-96. [PMID: 15706542]

32. Ditunno PL, Ditunno JF Jr. Walking index for spinal cord injury (WISCI II): Scale revision. Spinal Cord. 2001; 39(12):654-56. [PMID: 11781863]

33. Granger CV, Brownscheidle CM. Outcome measurement in medical rehabilitation. Int J Technol Assess Health Care. 1995;11(2):262-68. [PMID: 7790169]

34. Gresham GE, Labi ML, Dittmar SS, Hicks JT, Joyce SZ, Stehlik MA. The Quadriplegia Index of Function (QIF): Sensitivity and reliability demonstrated in a study of thirty quadriplegic patients. Paraplegia. 1986;24(1):38-44. [PMID: 3960588] 
35. Catz A, Itzkovich M, Steinberg F, Philo O, Ring H, Ronen J, Spasser R, Gepstein R, Tamir A. The Catz-Itzkovich SCIM: A revised version of the Spinal Cord Independence Measure. Disabil Rehabil. 2001;23(6):263-68.

[PMID: 11336099]

36. Marino RJ, Huang M, Knight P, Herbison GJ, Ditunno JF $\mathrm{Jr}$, Segal M. Assessing selfcare status in quadriplegia: Comparison of the Quadriplegia Index of Function (QIF) and the Functional Independence Measure (FIM). Paraplegia. 1993;31(4):225-33. [PMID: 8493037]

37. Catz A, Itzkovich M, Steinberg F, Philo O, Ring H, Ronen J, Spasser R, Gepstein R, Tamir A. Disability assessment by a single rater or a team: A comparative study with the
Catz-Itzkovich Spinal Cord Independence Measure. J Rehabil Med. 2002;34(5):226-30. [PMID: 12392238]

38. Law M, Baptiste S, McColl M, Opzoomer A, Polatajko H, Pollock N. The Canadian Occupational Performance Measure: An outcome measure for occupational therapy. Can J Occup Ther. 1990;57(2):82-87. [PMID: 10104738]

39. Carswell A, McColl MA, Baptiste S, Law M, Polatajko H, Pollock N. The Canadian Occupational Performance Measure: A research and clinical literature review. Can J Occup Ther. 2004;71(4):210-22. [PMID: 15586853]

Submitted for publication August 15, 2005. Accepted in revised form January 17, 2006. 
